\title{
New classes of spectral densities for lattice processes and random fields built from simple univariate margins
}

\author{
Emilio Porcu $\cdot$ Jorge Mateu $\cdot$ Pablo Gregori · \\ Martin Ostoja-Starzewski
}

Published online: 14 March 2012

(C) Springer-Verlag 2012

\begin{abstract}
Quasi arithmetic and Archimedean functionals are used to build new classes of spectral densities for processes defined on any $d$-dimensional lattice $\mathbb{Z}^{d}$ and random fields defined on the $d$-dimensional Euclidean space $\mathbb{R}^{d}$, given simple margins. We discuss the mathematical features of the proposed constructions, and show rigorously as well as through examples, that these new classes of spectra generalize celebrated classes introduced in the literature. Additionally, we obtain permissible spectral densities as linear combinations of quasi arithmetic or Archimedean functionals, whose associated correlation functions may attain negative values or oscillate between positive and negative ones. We finally show that these new classes of spectral densities can be used for nonseparable processes that are not necessarily diagonally symmetric.
\end{abstract}

Keywords Archimedeanity - Lattice processes · Nonseparability · Quasi arithmetic functionals · Random fields $\cdot$ Spectral densities

\footnotetext{
E. Porcu $(\square)$

University of Göttingen, Institut für Mathematische Stochastik, Göttingen, Germany

e-mail: eporcu@uni-goettingen.de

J. Mateu · P. Gregori

Department of Mathematics, Universitat Jaume I,

Castellón, Spain

J. Mateu · P. Gregori

IMAC Institut Universitari de Matemtiques i Aplicacions de

Castelló, Castellón, Spain

M. Ostoja-Starzewski

Department of Mechanical Science and Engineering, Institute

for Condensed Matter Theory, University of

Illinois at Urbana-Champaign, Urbana 61801, USA
}

\section{Introduction}

Modern mechanics of materials is largely driven by multiscale problems. The label under which much is done is called homogenization, where a deterministic approach is tacitly adopted. This absence of statistics stands in stark contrast to the recognition that most of heterogeneous matter is disordered and does not display any spatial periodicity. As a result, homogenization has to be stochastic in character, whereby one is interested in resolution of phenomena on length scales smaller than the so-called representative volume element (RVE) and/or problems lacking a separation of scales, e.g. (Ostoja-Starzewski 2002, 2008). The RVE should then be replaced by a statistical volume element (SVE) and a deterministic (usually homogeneous) field of material properties is replaced by a random field (RF). The SVE plays the role of a mathematical point in a macroscale problem. What follows next is either a strict-sense stationary (SSS) or a wide-sense stationary (WSS) model, the actual choice being dictated by the extent of available information. In the second case, one needs to set up a correlation function. A separate area of continuum physics where such functions are needed is turbulence, where the velocity field is also represented by WSS models, e.g. (Rytov et al. 1989). Another setting of RFs than the continuum-type models is offered by lattice-type systems, where the lattice is a set of inclusions in a composite material with a square-type micro-geometry but material randomness occurring on each lattice site. These are so-called lattice processes (LPs). Also here the first model one would consider is a WSS LP, for which a correlation function is required. It may well happen that in both cases - the continuum and the discrete one-the geometry of microstructure is not only random but also fractal. An extension of continuum thermomechanics in that direction has been taken in (OstojaStarzewski 2007). The correlation functions developed in this 
paper could be used to formulate thermomechanics on LPs. The construction of new classes of correlation functions and spectral densities for LPs or RFs is also crucial to geostatistics (Matheron 1989), and early literature has persistently emphasized the need for new models that allow for generality and desirable mathematical and statistical properties. In geostatistics the lattice is typically defined by the observer as a square grid on the surface being analyzed. Clearly, in both the lattice and the continuum case, having a good library from which to choose such functions is crucial, and further developing such a library is the task undertaken in this article.

As pointed out in Whittle (1954), a lattice or RF model can be built by starting from either a simple stochastic representation, a simple correlation structure or even a simple spectral density function. Considerable difficulties arise whenever the modeling approach is oriented to a global picture, that is, specifying all the three structures. This is confirmed for instance, by the literature on planar lattices, that finds a taxonomy in the celebrated categories of SAR (simultaneous autoregressive), CAR (conditional autoregressive; see Besag 1974) and unilateral ARMA models, and all these models, with exception to Martin (1996), have been proposed for the bidimensional case only. There are problems with CAR (and SAR) processes, as their covariance structures, as well as the elements of their inverse variance matrices corresponding to both sites on the boundary, are very difficult to obtain.

In the light of these difficulties, it seems reasonable to choose one of the three possible modeling strategies indicated before, knowing the tradeoff between them. In this article, we propose new classes of nonseparable spectral densities for either processes defined on infinite regular lattices of $\mathbb{Z}^{d}$ or RFs defined on $\mathbb{R}^{d}$. We shall show that our approach allows for building nonseparable spectral structures starting from simple margins, and sometimes permits to specify the corresponding correlation function, although the associated stochastic representation is all but trivial.

Our choice is motivated by the fact that a nonnegligible part of the (spatial) statistical community is interested in the specification of new models of spectral densities for nonseparable processes (Gneiting et al. 2007). Spectral techniques have been increasingly used, mainly in the last 10 years, for modeling LPs as well as RFs, for several reasons and advantages that both mathematical and statistical communities have persistently emphasized. Working in the spectral domain may simplify estimating procedures when using specified classes of correlation functions (Stein 1999). It also allows for testing for nonstationarity (Fuentes 2005), axial or diagonal symmetry (Scaccia and Martin 2005), space-time separability (Fuentes 2006), and for establishing important results on infill asymptotics with respect to the equivalence of kriging interpolators (Yadrenko 1983; Stein 1999; Zhang 2004). Some recent theoretical results strongly encourage the use of spectral techniques for spatial data: in his tour de force, Stein (1999) emphasizes the importance of the Matérn model for both spatial and spectral analysis, and justifies the effects of miss-specification of the spectral density, working on infill asymptotics. On the other hand, Fuentes (2002) studies the asymptotic properties of the periodogram via shrinking asymptotics, under the assumptions of either stationarity or nonstationarity. Working on fixed-domain asymptotics, Stein (1995) showed that standard asymptotic results for the periodograms do not apply, and the use of the raw data may yield misleading results, so that data tapers are needed.

Nonseparability is a crucial aspect for spatial modeling and several authors, in the Geostatistical context (Christakos 1991, 1992; Gneiting 2002; Stein 2005), as well as in the lattice framework (see Martin 1996, and references therein) have emphasized the limit of separable models in terms of behavior of the associated Best Linear Unbiased Predictor. At the same time, separable models have been very popular for their simplicity of construction, and for the considerable computational gains when dealing with large spatial datasets.

A natural question is then: given $d$ univariate margins, is there a nontrivial way to use them in order to build a nonseparable spectral density for LPs or RFs?

In this article, we propose quasi arithmetic (Kolmogorov 1930) and Archimedean functionals and use them to build new models of nonseparable spectral densities defined on any $d$-dimensional regular lattice or Euclidean space, given a number of margins. We show that this procedure allows for a wide class of spectral densities that admit as a special case the separable one. Also, we establish the mathematical properties of these constructions and discuss several examples that generalize existing classes of spectral densities or integrate the literature with new ones. In particular, both constructions give rise to families that are in general nonseparable and, additionally, quasi arithmetic functionals allow for building spectral densities that are not diagonally symmetric.

A first idea of using given margins to create a nonseparable structure can be found in Ma (2004), where a very simple model is obtained through the linear combination of the product of univariate correlation functions, that is $\rho(\mathbf{s} ; \alpha)=\theta \prod_{i=1}^{d} \rho_{i}\left(s_{i} ; \alpha_{i}\right)+(1-\theta) \prod_{i=1}^{d} \rho_{i}\left(s_{i} ; \beta_{i}\right), \quad \mathbf{s} \in \mathcal{S}$,

with $\alpha=\left(\alpha_{i}\right)_{i=1}^{d}, \mathcal{S}$ either the infinite rectangular lattice $\mathbb{Z}^{d}$ or the Euclidean space $\mathbb{R}^{d}$, and where the univariate correlation functions can be selected from univariate parametric families $\rho(\cdot ; \alpha)$ having desirable properties, such as the ARMA correlation functions for the lattice case or the Whittle-Matérn one (Matérn 1960) for the continuous one. Observe that the nonseparability of this model is solely 
determined by the parameter $\theta$, for which the author shows that this parameter can preserve the permissibility of the correlation structure even if belonging to a specified interval that is larger than the convex interval $[0,1]$.

This approach is important for several reasons. First, it allows for constructing models on any $d$-dimensional lattice or Euclidean space. Second, it is a very simple model for both correlation functions and associated spectral densities, viz.

$f(\omega ; \alpha)=\theta \prod_{i=1}^{d} f_{i}\left(\omega_{i} ; \alpha_{i}\right)+(1-\theta) \prod_{i=1}^{d} f_{i}\left(\omega_{i} ; \beta_{i}\right), \quad \omega \in \Omega^{d}$,

where $\Omega$ is either $[0, \pi]$ or the Euclidean space $\mathbb{R}$. Another general case, similar to this one, is treated in Gregori et al. (2008).

This construction gives an additional, important motivation to this paper. Ma's (2004) construction finds an intimate connection with the problem of negative correlation functions. As pointed out in Janauer (2001), there is a strong interaction between biology and hydrology in the establishment, fluctuation and limitation of the aquatic environment in space and time. For instance, the analysis of the spatio-temporal distribution of flow is strongly related to the study of current velocities and turbulences. In the spatial framework, Shkarofsky (1968) emphasized the fact that, in the study of turbulences it is often desirable to have covariance models allowing for negative values or oscillations from positive to negative values as the Euclidean distance tends to infinite. Unfortunately, most of previously proposed spatial covariance models in literature are positive in the whole domain, so they are not useful for this purpose.

The model in Eq. 1 allows to build correlation functions that may be negative or oscillate between negative and positive values. Thus, it seems natural to build models that generalize this construction and allow for establishing desirable mathematical-statistical properties and that can be used in many problems of physical, biological or environmental nature for which space time analysis is needed, such as in Pomeroy et al. (2003).

We shall show throughout the article that quasi arithmetic and Archimedean functionals allow for a nontrivial generalization of the model in Eq. 2), and thus allow for nonseparable structures whose Fourier pair may attain negative values.
In summary, our paper focuses on second-order stochastic models for geostatistical processes (see for example $\mathrm{Yu}, \mathrm{H}-\mathrm{L}$. et al. 2006), expressed through the spectrum density instead of the direct covariance function. We can build nonseparable structures under this methodology [see for example Porcu et al. (2006, (2008) ], starting from well-known margin models that we can assemble through some simple and easily interpretable link functions. Data from many environmental geostatistical processes show thoroughly the need of simple and flexible nonseparable models. We propose these models for their simplicity in the way the model is built up, and the flexibility provided by the many choices of margins and link functions. These combinations of marginal functions to provide multivariate versions have been used in spatio-temporal wavelet analysis (Ruiz-Medina and Angulo 2002).

The remainder of the article is organized as follows: in Sect. 2 basic facts about RFs or LPs, correlation functions and spectral densities are presented. Section 3 introduces the new classes of spectral densities and discusses its features, the associated permissibility criteria and some instructive examples. In Sect. 4, some special permissibility criteria are deduced for linear combinations of these new classes of spectra, when the parameters in the linear combinations are set to be negative. Section 5 concludes the article with some discussion.

\section{Preliminaries}

Consider a zero mean weakly stationary real-valued process $\{Z(\mathbf{x}), \mathbf{x} \in \mathcal{S}\}$, for $\mathcal{S}$ either the lattice $\mathbb{Z}^{d}$ or the continuum $\mathbb{R}^{d}$. For convenience, it is assumed that the process is Gaussian, although usually only second-order properties are used. For $\mathbf{x}, \mathbf{y} \in \mathcal{S}$, let $\mathbf{x}-\mathbf{y}=\mathbf{s}=\left(s_{1}, \cdots, s_{d}\right) \in \mathcal{S}$ be a vector of lags; the corresponding correlation function $\rho: \mathcal{S} \rightarrow \mathbb{R}$ is defined as

$\rho(\mathbf{s})=\frac{\mathbb{E}(Z(\mathbf{s}) Z(\mathbf{0}))}{\mathbb{E} Z(\mathbf{0})^{2}}, \quad \mathbf{s} \in \mathcal{S}$.

Recall that, by Bochner's theorem (1933), correlation functions are positive definite, that is, the class of correlation functions coincides with that of Fourier transforms of probability measures. Assuming that the corresponding measure is Lebesgue-absolutely continuous, the Fourier pair associated to $\rho(\cdot)$, called spectral density, is defined as

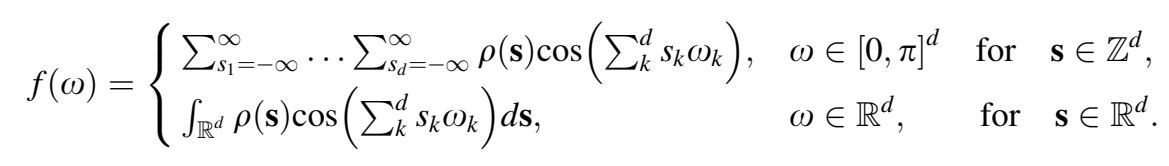


with $\omega=\left(\omega_{1}, \ldots, \omega_{d}\right)$ and $\mathbf{s}=\left(s_{1}, \ldots, s_{d}\right)$. For construction, $f$ must be nonnegative and absolutely integrable on its domain $\Omega^{d}$ (denoted with $f \in L_{+}^{1}\left(\Omega^{d}\right)$ ), with $\Omega$ either $[0, \pi]$ or $\mathbb{R}$.

A spectral density is called axially symmetric if $f\left(\omega_{1}, \ldots, \omega_{i}, \ldots, \omega_{d}\right)=f\left(\omega_{1}, \ldots,-\omega_{i}, \ldots, \omega_{d}\right)$ for every $i=1, \ldots, d$. It is diagonally symmetric if it is invariant under permutation of the arguments, that is $f\left(\omega_{1}, \ldots\right.$, $\left.\omega_{i}, \ldots, \omega_{j}, \ldots, \omega_{d}\right)=f\left(\omega_{1}, \ldots, \omega_{j}, \ldots, \omega_{i}, \ldots, \omega_{d}\right)$, for every $i, j=1, \ldots, d$. Spectra that are both axially and diagonally symmetric are called fully symmetric. In the Geostatistical framework, it is very popular to set spectral densities as functions of the Euclidean norm, that is $f(\omega):=\widetilde{f}(\|\omega\|)$. In this case, spectral densities are invariant under rotation, and are commonly called isotropic.

Several models of spectral densities can be found in the literature. In the following, we list some models that we use in the examples throughout the article. As for the lattice case, consider $\{Z(t), t \in \mathbb{Z}\}$ a stationary $\operatorname{ARMA}(p, q)$ process generated from the stochastic difference equation

$$
\begin{aligned}
& \left(1-\alpha_{1} B\right) \cdots\left(1-\alpha_{p} B\right) Z(t)=\left(1-\alpha_{p+1} B\right) \cdots \\
& \quad \times\left(1-\alpha_{p+q} B\right) \epsilon(t),
\end{aligned}
$$

for $\quad-1<\alpha_{k}<1, k=1, \cdots, p+q, \quad$ constants, $B \quad$ the backwards shift operator, and $\{\epsilon(t), t \in \mathbb{Z}\}$ a standard white noise. The corresponding spectral density is

$$
\begin{gathered}
f_{\mathrm{ARMA}}(\omega ; \alpha)=\frac{\prod_{k=1}^{q}\left(1-2 \alpha_{p+k} \cos \omega+\alpha_{p+k}^{2}\right)}{\prod_{k=1}^{p}\left(1-2 \alpha_{k} \cos \omega+\alpha_{k}^{2}\right)}, \\
\omega \in[0, \pi] .
\end{gathered}
$$

A separable ARMA LP is simply built through the tensorial product of univariate ARMA spectral densities or correlation functions. This technique was first proposed by Martin (1996). Observe that an ARMA (1,0) is simply an autoregressive process of the first order $(\operatorname{AR}(1))$, and $\mathrm{AR}(1) \times \mathrm{AR}(1)$ models have been widely used in the literature (cfr. Scaccia and Martin 2005, and references therein).

For the continuous case, an interesting class is that of continuous $\operatorname{AR}(p)$ models, satisfying the stochastic differential equation

$Z(t)+\phi_{1} Z^{\prime}(t)+\cdots+\phi_{p} Z^{(p)}(t)=\varepsilon^{\prime}(t)$,

whose spectral density factorizes as

$f_{\mathrm{AR}}(\omega ; \alpha)=\prod_{i=1}^{p} \frac{\alpha_{i}}{\alpha_{i}^{2}+\omega^{2}}$,

for distinct constants $\alpha_{i}>0$ and $\{\varepsilon(t), t \in \mathbb{R}\}$ a standard Brownian motion. Another spectral density that can be useful in this context is the one associated to the isotropic Matérn covariance function on $\mathbb{R}^{d}$, having expression

$f_{\text {Mat }}(\omega ; \alpha, v) \propto \alpha^{2 v}\left(\alpha^{2}+\|\omega\|^{2}\right)^{-v-d / 2}, \quad \omega \in \mathbb{R}^{d}$

for constants $\alpha, v>0$. The associated process is $[v]-1$ times mean-square differentiable, where $[v]$ is the largest integer less than or equal to $v$.

\section{Archimedean and quasi arithmetic constructions}

\subsection{Axiomatica}

Let $(\Omega, \mu)$ be the Lebesgue measure space where $\Omega$ can be chosen to be either $[0, \pi]$ or $\mathbb{R}$. For an integer $k>1$, let $\left(d_{i}\right)_{i=1}^{k}$ be $k$ positive integers, and $d=\sum_{i=1}^{k} d_{i}$. For simplicity, let us denote $\mathcal{F}:=\prod_{i=1}^{k} L_{+}^{1}\left(\Omega^{d_{i}}\right)$ the space of vectors of $k$ nonnegative integrable functions defined in the corresponding spaces, and its elements denoted as $\boldsymbol{f}=\left(f_{i}\right)_{i=1}^{k}$, with $\sum_{i} d_{i}=d, d \geq 2$. Observe that a vector of $k$ spectral densities belong to $\mathcal{F}$. Finally, let us express each vector $\omega \in \Omega^{d}$ as $\omega=\left(\omega_{1}, \ldots, \omega_{k}\right)$ where $\omega_{i} \in \Omega^{d_{i}}$ for $i=1, \ldots, k$. Let $\Phi$ be the class

$\Phi=\{\varphi:(0, \infty) \rightarrow \mathbb{R}: \varphi$

strictly monotonic and continuous $\}$.

For $\varphi \in \Phi$, the functional equations

$\varphi(f(\omega))=\sum_{i=1}^{k} \varphi\left(f_{i}\left(\omega_{i}\right)\right), \quad \omega \in \Omega^{d}$,

and

$\varphi(f(\omega))=\sum_{i=1}^{k} \mathrm{w}_{i} \varphi\left(f_{i}\left(\omega_{i}\right)\right), \quad \omega \in \Omega^{d}$,

with $\sum_{i} \mathrm{w}_{i}=1, \mathrm{w}_{i} \geq 0$, have been proposed for different purposes and in several fields of mathematics and statistics. Their solutions are, respectively,

$f(\omega)=\varphi^{-1}\left(\sum_{i=1}^{k} \varphi\left(f_{i}\left(\omega_{i}\right)\right)\right):=\mathcal{A}_{\varphi}(\boldsymbol{f})(\omega)$,

and

$f(\omega)=\varphi^{-1}\left(\sum_{i=1}^{k} \mathrm{w}_{i} \varphi\left(f_{i}\left(\omega_{i}\right)\right)\right):=M_{\varphi}(\boldsymbol{f})(\omega)$.

Although these solutions seem to be very similar, we shall see in the sequel that they have different impacts on the conditions for the function $\varphi$ in order to ensure permissibility (viz., absolute integrability) of the resulting structures in Eqs. 6 and 
7, as well as for the mathematical properties of these compositions, as it is shown subsequently.

Remark 3.1 Let $I \subset \mathbb{R}$ be an interval, and let $\varphi: I \rightarrow \mathbb{R}$ belong to $\Phi$. Then $\mathcal{A}_{\psi}=\mathcal{A}_{\varphi}$ and $M_{\psi}=M_{\varphi}$ if and only if $\psi(x)=a_{1} \varphi(x)+a_{2}, \quad x \in I$,

for some $a_{1}, a_{2} \in \mathbb{R}, a_{1} \neq 0$. Thus, we can characterize $\varphi \in$ $\Phi$ in terms of monotonically decreasing functions.

This remark allows us to restrict, for the remainder of the paper, to the class of continuous strictly decreasing functions without loss of generality.

The functional $\mathcal{A}_{\varphi}$ is called Archimedean and the solution of the functional equation in (6), Archimedean composition. These compositions are familiar to those working with copulas (Genest 1987; Genest and MacKay 1986), that is, multivariate distributions with fixed margins. The construction of nonstationary covariance functions by the original technique of spatial adaption of spectra (Pintore and Holmes 2004) has been extended using a similar Archimedean composition (Porcu et al. 2009). These authors first use the concept of Archimedean composition in a different framework, oriented to the construction of nonstationary covariances for spatial data and under the restrictive assumption of isotropy. The framework proposed herein is completely general and a characterization of Archimedean composition is given. One can notice that these results are independent of those presented in Porcu et al. (2009). It is worth remarking that permissibility conditions are clearly different if the composition is either Archimedean or quasi arithmetic, and this is the aspect we are stressing repeatedly throughout this article.

Let us call $\varphi$ (or equivalently its proper inverse) the generator of the Archimedean composition. Archimedean compositions admit as a special case the tensorial product of spectral densities, that is $\mathcal{A}_{\Pi}(\boldsymbol{f})(\omega)=\prod_{i=1}^{k} f_{i}\left(\omega_{i}\right)$, that corresponds to the case of separability as discussed in Martin (1996). In this case the generator is the completely monotonic mapping $t \mapsto \varphi^{-1}(t)=\exp (-t)$.

The functional $M_{\varphi}$ is called quasi arithmetic, and thus Eq. 7 defines a weighted quasi arithmetic mean of the involved spectral densities, called margins. The function $\varphi$, or its proper inverse is commonly known as generator of the quasi arithmetic mean. Quasi arithmetic operators have been extensively treated in Hardy et al. (1934) even if Kolmogorov (1930) and Nagumo (1930) derived first, and independently of each other, necessary and sufficient conditions for the quasi arithmeticity of a mean. Using this result, they partially modified the classical Cauchy (1821) internality and Chisini's (1929) invariance properties. As pointed out by Marichal (2000), the Kolmogorov reflexive property is equivalent to the Cauchy internality, and both are accepted by statisticians as requisites for means. Here, our main idea is to use the properties of this class of operators in order to create permissible classes of spectral densities that attain desirable mathematical features. For a vector of real numbers $\xi=\left(\xi_{i}\right)_{i=1}^{k}$, well known properties of quasi arithmetic operators are:

1. Internality, that is $\min _{i}\left(\xi_{i}\right) \leq M_{\varphi}(\xi) \leq \max _{i}\left(\xi_{i}\right)$;

2. $\varphi$ convex implies $M_{\varphi}(\xi) \leq \sum_{i=1}^{k} \mathrm{w}_{i} \xi_{i}$;

3. The class generated by $\varphi_{r}(t)=t^{r}, r \in \mathbb{R} \backslash\{0\}$, includes the harmonic mean (dubbed $M_{H}, r=-1$ ), the arithmetic mean (dubbed $M_{\Sigma}, r=1$ ), and the degenerated cases: "minimum" value $(r \rightarrow-\infty)$, the geometric mean (dubbed $M_{\Pi}, r \rightarrow 0$ ), and the "maximum" value $(r \rightarrow+\infty)$. This class of means is called homogeneous, as $M_{\varphi}(a \xi)=a M_{\varphi}(\xi), \forall a \in \mathbb{R}$.

4. The following pointwise orderings can be deduced (Hardy et al. 1934): for $\varphi_{1}, \varphi_{2} \in \Phi$,

(a) If $\varphi_{1} \circ \varphi_{2}^{-1}$ is convex (resp. concave), then $M_{\varphi_{1}}(\xi) \leq M_{\varphi_{2}}(\xi)($ resp. $\geq)$

(b) Additionally, for $\varphi$ convex,

$\min _{i}\left(\xi_{i}\right) \leq M_{H}(\xi) \leq M_{\varphi}(\xi) \leq M_{\Pi}(\xi) \leq M_{\Sigma}(\xi) \leq \max _{i}\left(\xi_{i}\right)$.

It is worth mentioning that Archimedean functionals do not possess the properties of mean operators, as they do not satisfy the internality property in the sense of Chisini (1929). However, we can present in the next result the ordering relations for the quasi arithmetic mean operators with some other ones that can be stated for the Archimedean ones. For simplicity of notation, let us use the order relation symbols, $\leq$ and $\geq$, among functions, whenever the corresponding order relation holds for all $\omega \in \Omega^{d}$ (and taking into account the convention $\infty \leq \infty$ ).

Corollary 3.2 Let $\varphi_{1}, \varphi_{2} \in \Phi, \boldsymbol{f} \in \mathcal{F}$. Let $\mathcal{A}_{\varphi_{i}}$ and $M_{\varphi_{i}}$ as defined in Eqs. 6 and 7, respectively. Then:

(i) If $\varphi_{1} \circ \varphi_{2}^{-1}$ is subadditive (resp. superadditive), then $\mathcal{A}_{\varphi_{1}}(\boldsymbol{f}) \leq \mathcal{A}_{\varphi_{2}}(\boldsymbol{f})($ resp. $\geq$ ).

(ii) In particular, if $\varphi \circ \exp$ is subadditive (resp. superadditive), then $\mathcal{A}_{\varphi}(\boldsymbol{f}) \leq \mathcal{A}_{\Pi}(\boldsymbol{f})($ resp $\geq$ ).

(iii) If $\varphi_{1} \circ \varphi_{2}^{-1}$ is convex (resp. concave), then $M_{\varphi_{1}}(\boldsymbol{f}) \leq$ $M_{\varphi_{2}}(\boldsymbol{f})($ resp. $\geq)$.

(iv) For $\varphi$ convex on $[0, \infty)$,

$\min (\boldsymbol{f}) \leq M_{H}(\boldsymbol{f}) \leq M_{\varphi}(\boldsymbol{f}) \leq M_{\Pi}(\boldsymbol{f}) \leq M_{\Sigma}(\boldsymbol{f}) \leq \max (\boldsymbol{f})$.

where $\min (\boldsymbol{f})(\omega)=\min _{i}\left(f_{i}\left(\omega_{i}\right)\right)$ and $\max (\boldsymbol{f})(\omega)=\max _{i}$ $\left(f_{i}\left(\omega_{i}\right)\right)$.

Some comments are in order. Corollary 3.2 gives important information on the LP or RF in terms of variances. For instance, the variance of the associated RF will be $\sigma^{2}=\mathrm{C}(\boldsymbol{0})=\int_{\omega \in \mathbb{R}^{d}} M_{\varphi}(\boldsymbol{f})(\omega) \mathrm{d} \omega$. Thus, under the relevant 
conditions in Corollary 3.2, we know the corresponding ordering, in terms of variances, of either the associated LPs or the RFs. Additionally, in the last case, some more information can be obtained with respect to the mean square (m.s.) differentiability of the associated RF, that is linked to the spectral density by the following well-known identity: $Z$ is m.s. differentiable of order $v$ if and only if the spectral moments of order $2 v$ exist and are finite. Thus, finite spectral moment of the margins $f_{i}$, ensures the finite spectral moment of associated composition $M_{\varphi}(\boldsymbol{f})$ and this has an immediate consequence on the m.s. differentiability of the associated RF.

\subsection{Permissibility criteria for $\mathcal{A}_{\varphi}(\boldsymbol{f})$ and $M_{\varphi}(\boldsymbol{f})$}

As far as the integrability of these functions is not trivial, for each $f \in \mathcal{F}$ we denote with $\Phi_{f}^{\mathcal{A}}$ (resp. $\Phi_{f}^{M}$ ), the subset of $\Phi$ of functions $\varphi$ for which $\mathcal{A}_{\varphi}(\boldsymbol{f})$ (resp. $\left.M_{\varphi}(\boldsymbol{f})\right)$ is integrable in $\Omega^{d}$. For each $\mathcal{G} \subset \mathcal{F}$ we write $\Phi_{\mathcal{G}}^{\mathcal{A}}=\bigcap_{f \in \mathcal{G}} \Phi_{f}^{\mathcal{A}}$ (resp. $\Phi_{\mathcal{G}}^{M}=\bigcap_{f \in \mathcal{G}} \Phi_{f}^{M}$ ).

\subsubsection{The lattice case}

Here, integrability of quasi arithmetic and Archimedean compositions is ensured by construction, hence $\Phi_{\mathcal{F}}^{\mathcal{A}}=\Phi_{\mathcal{F}}^{M}=$ $\Phi$. Being $\varphi$ decreasing on the positive real line, it is, automatically, subadditive. Thus, for $\Omega=[0, \pi]$,

$$
\begin{gathered}
\int_{\Omega^{d}} \mathcal{A}_{\varphi}(\boldsymbol{f})(\omega) d \omega \leq \int_{\Omega^{d}} \sum_{i=1}^{k} f_{i}\left(\omega_{i}\right) d \omega=\sum_{i=1}^{k}\left[\prod_{j \neq i} \mu\left(\Omega^{d_{j}}\right)\right] \\
\int_{\Omega^{d_{i}}}\left|f_{i}\left(\omega_{i}\right)\right| d \omega_{i}=\sum_{i=1}^{k} \mu(\Omega)^{d-d_{i}} \int_{\Omega^{d_{i}}}\left|f_{i}\left(\omega_{i}\right)\right| d \omega_{i} .
\end{gathered}
$$

On the other hand, under the assumption of weak stationarity, spectral densities are always bounded (by the integral of the absolute value of the covariance function). Thus, we get

$$
\int_{\Omega^{d}} M_{\varphi} \boldsymbol{f}(\omega) d \omega \leq \int_{\Omega^{d}} \max _{i} f_{i}\left(\omega_{i}\right) d \omega \leq \mu\left(\Omega^{d}\right) \max _{i} \max _{\omega_{i} \in \Omega_{i}}\left|f_{i}\left(\omega_{i}\right)\right| .
$$

\subsubsection{The RF case}

Here, much more caution is needed depending either on the generator or on the spectral densities involved in the quasi arithmetic or Archimedean compositions. To this purpose, we shall restrict ourselves to $\mathcal{F}_{(A)}$ the subset of $\mathcal{F}$ of vectors of functions $f$ satisfying the following condition:

\subsubsection{Condition (A)}

There exists some $\gamma_{i} \in \mathbb{R}^{+}$for $i=1, \ldots, k$ such that $f_{i}\left(\omega_{i}\right)=O\left(\left\|\omega_{i}\right\|^{-\gamma_{i}}\right)$ for $\left\|\omega_{i}\right\| \rightarrow \infty$ and all $i=1,2, \ldots, k$, and there exists at least one $j \in\{1, \ldots, k\}$ such that $f_{j}\left(\omega_{j}\right)=O(1)$ for $\omega_{j} \rightarrow 0$.

Obviously, spectral densities are bounded at the origin, and many well known examples fulfill condition (A). Now we show the following result, useful for obtaining interesting examples.

Proposition 3.3 For $\Omega=\mathbb{R}$, we have that:

(i) $t \mapsto-\log (t) \in \Phi_{\mathcal{F}}^{\mathcal{A}}$.

(ii) $t \mapsto(1-t)_{+} \in \Phi_{\mathcal{F}}^{\mathcal{A}}$.

(iii) $t \mapsto t^{-1 / \varepsilon} \in \Phi_{\mathcal{F}(A)}^{\mathcal{A}}$ for any $\varepsilon>0$, whenever $\sum_{i=1}^{k} \frac{d_{i}}{\gamma_{i}}<$ 1.

(iv) $\varphi \in \Phi_{\mathcal{F}(A)}^{M}$ whenever $\varphi$ is convex and $\frac{d_{i}}{\gamma_{i}}<\mathrm{w}_{i}$ for each $i=1, \ldots, k$.

Proof (i) and (ii) are easy to prove. For (iii) and (iv) we use the sign $\sim$ to express "the same character of integrability" among the integrals in the following lines, and use typical changes of variables to proceed along the proof. For the point (iii), notice that

$$
\begin{aligned}
& \int_{\mathbb{R}^{d}} \mathcal{A}_{\varphi}(\boldsymbol{f})(\omega) \mathrm{d} \omega=\int_{\mathbb{R}^{d_{1}}} \cdots \int_{\mathbb{R}^{d_{k}}}\left(\sum_{i=1}^{k} f_{i}\left(\omega_{i}\right)^{-1 / \varepsilon}\right)^{-\varepsilon} \mathrm{d} \omega_{1} \cdots \mathrm{d} \omega_{k} \\
& \sim \int_{\left\|\omega_{1}\right\|>1} \ldots \int_{\left\|\omega_{k}\right\|>1}\left(\sum_{i=1}^{k}\left\|\omega_{i}\right\|^{\gamma_{i} / \varepsilon}\right)^{-\varepsilon} \mathrm{d} \omega_{1} \cdots \mathrm{d} \omega_{k} \\
& \sim \int_{r_{1}>1} \ldots \int_{r_{k}>1}\left(\sum_{i=1}^{k} r_{i}^{\gamma_{i} / \varepsilon}\right)^{-\varepsilon}\left(\prod_{i=1}^{k} r_{i}^{d_{i}-1}\right) \mathrm{d} r_{1} \cdots \mathrm{d} r_{k} \\
& \sim \int_{t_{1}>1} \ldots \int_{t_{k}>1}\left(\sum_{i=1}^{k} t_{i}^{2}\right)^{-\varepsilon}\left(\prod_{i=1}^{k} t_{i}^{\frac{2 \varepsilon d_{i}}{\gamma_{i}}}-1\right) \mathrm{d} t_{1} \cdots \mathrm{d} t_{k} \\
& \sim \int_{\rho>1} \rho^{-2 \varepsilon-1+\sum_{i=1}^{k} \frac{2 \varepsilon d_{i}}{\gamma_{i}}} \mathrm{~d} \rho .
\end{aligned}
$$

Therefore the inequality of (iii) follows directly from integrability of the last expression.

Point (iv) is simpler since any convex $\varphi$ implies the boundedness $M_{\varphi}(\boldsymbol{f})(\omega) \leq \prod_{i=1}^{k} f_{i}\left(\omega_{i}\right)^{\mathrm{w}_{\mathrm{i}}} \quad$ (the geometric mean), hence the separate inequalities of (iv) are obtained from the last of the following integrals: 


$$
\begin{aligned}
& \int_{\mathbb{R}^{d_{1}}} \cdots \int_{\mathbb{R}^{d_{k}}} \prod_{i=1}^{k} f_{i}\left(\omega_{i}\right)^{\mathrm{w}_{\mathrm{i}}} \mathrm{d} \omega_{1} \cdots \mathrm{d} \omega_{k}=\prod_{i=1}^{k} \int_{\mathbb{R}^{d_{i}}} f_{i}\left(\omega_{i}\right)^{\mathrm{w}_{\mathrm{i}}} \mathrm{d} \omega_{i} \\
& \sim \prod_{i=1}^{k} \int_{\left\|\omega_{i}\right\|>1}\left\|\omega_{i}\right\|^{-\mathrm{w}_{\mathrm{i}} \gamma_{\mathrm{i}}} \mathrm{d} \omega_{i} \\
& \sim \prod_{i=1}^{k} \int_{r_{i}>1} r_{i}^{-\mathrm{w}_{\mathrm{i}} \gamma_{\mathrm{i}}+\mathrm{d}_{\mathrm{i}}-1} \mathrm{~d} r_{i} .
\end{aligned}
$$

Remark 3.4 The nonnegativity of the quasi arithmetic and Archimedean spectral densities is ensured by contruction, but this aspect will be a crucial point for Sect. 4, where we discuss the case of linear combinations of quasi arithmetic or Archimedean spectra with negative weights.

Example 3.5 Let us give examples for (a) the lattice case and (b) the continuous one.

(a) For LPs, we can take any decreasing $\varphi \in \Phi$, independently of the vector of functions $\boldsymbol{f}$ representing the margins. For instance, both the expressions

$\mathcal{A}_{\varphi}(\boldsymbol{f})(\omega)=\left(\sum_{i=1}^{k} f_{i}\left(\omega_{i}\right)^{-\varepsilon}\right)^{-1 / \varepsilon}$,

and

$M_{\varphi}(\boldsymbol{f})(\omega)=\left(\sum_{i=1}^{k} \mathrm{w}_{\mathrm{i}} f_{i}\left(\omega_{i}\right)^{-\varepsilon}\right)^{-1 / \varepsilon}$,

for $\left(\mathrm{w}_{\mathrm{i}}\right)_{i=1}^{k}$ nonnegative weights summing up to one, can be seen as either parametric or semiparametric families of spectral densities indexed by the parameter $\varepsilon>0$, where the semiparametric construction would derive from the choice of nonparametric margins. Otherwise, the structure would be fully parametric. Thus, the margins can be selected among the ones listed in Sect. 2, such as those of $\operatorname{ARMA}(p, q)$ type and in any case the permissibility of the resulting structure would be preserved.

(b) The continuous case needs caution in the choice of both generators and margins. In the case of Archimedean compositions, let the generator be the function $t \mapsto \varphi(t)=t^{-1 / \varepsilon}, \varepsilon>0$, and let the margins of the Matérn type as in Eq. 5. One can verify that the conditions needed on the margins in order to ensure the integrability of the resulting structure are $\sum_{i=1}^{k} \frac{d_{i}}{2 v_{i}+d_{i}}<1$. We thus get that, for $\eta_{i}=$ $\left(v_{i}+d_{i} / 2\right) / \varepsilon$,

$$
\mathcal{A}_{\varphi}(\boldsymbol{f})(\omega)=\left(\sum_{i=1}^{k} \alpha_{i}^{-2 v_{i} / \varepsilon}\left(\alpha_{i}^{2}+\left\|\omega_{i}\right\|^{2}\right)^{\eta_{i}}\right)^{-\varepsilon}
$$

is permissible.

In the case of quasi arithmetic operators, if $\frac{d_{i}}{2}\left(\frac{1}{\mathrm{w} i}+\right.$ 1) $<v_{i}$ for each $i=1, \ldots, k$, one can easily verify that the integrability of the geometric mean over $\Omega^{d}$ is ensured, and thus the class

$M_{\varphi}(\boldsymbol{f})(\omega)=\left(\sum_{i=1}^{k} \mathrm{w}_{i} \alpha_{i}^{-2 v_{i} / \varepsilon}\left(\alpha_{i}^{2}+\left\|\omega_{i}\right\|^{2}\right)^{\eta_{i}}\right)^{-\varepsilon}$,

for $\eta_{i}=\left(v_{i}+d_{i} / 2\right) / \varepsilon$, is permissible for any positive $\varepsilon$.

A counterexample of not-permissibility can be obtained by taking a composition of continuous $\operatorname{AR}(p)$ processes as defined in Eq. 4. Under the setting previously described, we get that the composition

$M_{\varphi}(\boldsymbol{f})(\omega)=\left(\sum_{i=1}^{k} \mathrm{w}_{i} \prod_{j=1}^{p}\left(\frac{\alpha_{j i}^{2}+\left\|\omega_{i}\right\|^{2}}{\alpha_{j i}}\right)^{1 / \varepsilon}\right)^{-\varepsilon}$

is not permissible, as can be easily verified from Proposition 3.3 .

\section{Linear combinations of Archimedean and quasi arithmetic spectra related to negative-valued covariance functions}

In the light of what has been shown in the previous section, we can now analyze the construction in Eq. 2, that is based on linear combinations of the product of univariate and parametric spectral densities. According to the notation used throughout the paper, this construction can now be rephrased as

$\theta \mathcal{A}_{\Pi}(\boldsymbol{f})(\omega)+(1-\theta) \mathcal{A}_{\Pi}(\boldsymbol{g})(\omega), \quad \omega \in \Omega^{d}$,

where $\Omega$ is either $[0, \pi]$ or the Euclidean space $\mathbb{R}$, and where $\boldsymbol{f}(\cdot)=\left(f_{1}\left(\cdot ; \alpha_{1}\right), \cdots, f_{k}\left(\cdot ; \alpha_{k}\right)\right)^{\prime} \quad$ and $\boldsymbol{g}(\cdot)=\left(g_{1}(\cdot), \cdots, g_{k}\right.$ $(\cdot))^{\prime}=\left(f_{1}\left(\cdot ; \beta_{1}\right), \cdots, f_{k}\left(\cdot ; \beta_{k}\right)\right)^{\prime}$ are vectors of spectral densities belonging to specified parametric families. It is quite evident that this construction can be generalized in two directions that can be sketched as follows: let us consider two vectors of (parametric or not) $k$ spectral densities $\boldsymbol{f}, \boldsymbol{g} \in \mathcal{F}$, and set two generators $\varphi_{1}, \varphi_{2} \in \Phi$.

The following linear combinations

$f_{\mathcal{A}}(\omega)=\theta \mathcal{A}_{\varphi_{1}} \boldsymbol{f}(\omega)+(1-\theta) \mathcal{A}_{\varphi_{2}} \boldsymbol{g}(\omega)$,

and

$f_{\mathrm{M}}(\omega)=\theta M_{\varphi_{1}} \boldsymbol{f}(\omega)+(1-\theta) M_{\varphi_{2}} \boldsymbol{g}(\omega)$,

for $\theta \in \mathbb{R}$, give a wider scenario that constitutes the natural generalization of (1.2) to the case of different generators, different operators, and to the margins that are not 
necessarily belonging to a parametric family as in $\mathrm{Ma}$ (2004). In this section, we are seeking the conditions for $f_{\mathcal{A}}$ and $f_{\mathrm{M}}$ to be permissible. The integrability of both linear combinations depends on the assumptions on the sets of spectral densities and on the generating functions, as shown in previous section. Now let us denote

$$
\begin{aligned}
q_{i}\left(\omega_{i}\right) & =\frac{f_{i}\left(\omega_{i}\right)}{g_{i}\left(\omega_{i}\right)}, \quad M_{i}=\sup _{\omega_{i} \in \Omega^{d_{i}}} q_{i}\left(\omega_{i}\right), \\
m_{i} & =\inf _{\omega_{i} \in \Omega^{d_{i}}} q_{i}\left(\omega_{i}\right),
\end{aligned}
$$

for $i=1,2, \ldots, k$, and let $q(\omega)$ be either

$\frac{M_{\varphi_{1}}(\boldsymbol{f})(\omega)}{M_{\varphi_{2}}(\boldsymbol{g})(\omega)} \quad$ or $\quad \frac{\mathcal{A}_{\varphi_{1}}(\boldsymbol{f})(\omega)}{\mathcal{A}_{\varphi_{2}}(\boldsymbol{g})(\omega)}$

depending on the considered linear combination. Finally, let us denote its extreme values by $M^{*}=\sup _{\omega \in \Omega^{d}} q(\omega)$ and $m^{*}=\inf _{\omega \in \Omega^{d}} q(\omega)$. We are now able to state the more general, abstract result, and then give some necessary comments on it.

Proposition 4.1 Provided that the functions $f_{\mathcal{A}}$ and $f_{\mathrm{M}}$ defined in Eqs. 10-11 are integrable, they are, respectively, valid spectral densities if and only if

$\left[1-\max \left(1, M^{*}\right)\right]^{-1} \leq \theta \leq\left[1-\min \left(1, m^{*}\right)\right]^{-1}$,

where conventions $0^{-1}=-\infty$ and $(-\infty)^{-1}=0$ for the left hand side, and $0^{-1}=+\infty$ for the right hand side, are adopted.

Proof Being the two terms of the combination separately nonnegative, their linear combination is nonnegative if and only if $\theta q(\omega)+(1-\theta) \geq 0$, hence if and only if $\theta(1-$ $q(\omega)) \leq 1$ for all $\omega \in \mathbb{R}^{d}$. The set of values of $\theta$ satisfying the previous inequality for all $\omega \in \Omega^{d}$ can be written as

$A \cap B$ :

$$
\begin{aligned}
= & \left(\bigcap_{\{\omega: q(\omega)<1\}}\left(-\infty,(1-q(\omega))^{-1}\right]\right) \\
& \cap\left(\bigcap_{\{\omega: q(\omega)>1\}}\left[(1-q(\omega))^{-1},+\infty\right)\right)
\end{aligned}
$$

where $A$ (resp. $B$ ) can be the trivial set $\mathbb{R}$ in case $q(\omega)>1$ (resp. $q(\omega)<1$ ) for all $\omega \in \Omega^{d}$.

Let us assume, without loss of generality, that $A$ and $B$ are both nontrivial (therefore that $0 \leq m^{*}<1<$ $\left.M^{*} \leq+\infty\right)$. Take two respective sequences $\left\{\omega_{k}^{m}\right\}_{k=1}^{\infty}$ and $\left\{\omega_{k}^{M}\right\}_{k=1}^{\infty}$ such that $q\left(\omega_{k}^{m}\right)<1$ for all $k,\left\{q\left(\omega_{k}^{m}\right)\right\}_{k}$ is decreasing and $\lim _{k \rightarrow \infty} q\left(\omega_{k}^{m}\right)=m^{*}$, and similarly, $q\left(\omega_{k}^{M}\right)>1$ for all $k,\left\{q\left(\omega_{k}^{M}\right)\right\}_{k}$ is increasing and $\lim _{k \rightarrow \infty} q\left(\omega_{k}^{M}\right)=M^{*}$. Then

$$
\begin{aligned}
A \cap B & =\left(\bigcap_{k=1}^{\infty}\left(-\infty,\left(1-q\left(\omega_{k}^{m}\right)\right)^{-1}\right]\right) \\
& \cap\left(\bigcap_{k=1}^{\infty}\left[\left(1-q\left(\omega_{k}^{M}\right)\right)^{-1},+\infty\right)\right) \\
& =\left(-\infty,\left(1-m^{*}\right)^{-1}\right] \cap\left[\left(1-M^{*}\right)^{-1},+\infty\right) \\
& =\left[\left(1-M^{*}\right)^{-1},\left(1-m^{*}\right)^{-1}\right] .
\end{aligned}
$$

Obviously, for each case of trivial set ( $A$ or $B$ equal to $\mathbb{R}$ ) the interval is unbounded by the respective side, leaving the notation of the interval used in the claim of the proposition rather compact.

Remark 4.2 Only if $M^{*}=+\infty$ and $m^{*}=0$, a valid spectral density of the form (10) or (11) must have both coefficients $\theta$ and $1-\theta$ nonnegative. Otherwise, (10) and (11) may be spectral densities with a negative weight, and hence with eventually negative values of the associated covariance functions.

This result, as it will be explained subsequently, is of difficult application, but it puts the basis for some special results that are of interest for spectral modeling. For practical applications, calculations of $M^{*}$ and $m^{*}$, which not only depend on the pair of sets of functions, but also on the way the Archimedean compositions or quasi arithmetic means combine such functions, are intractable: the analytic expression of the quotient $q(\omega)$ expands without cancellations (except in the case of the tensor product, as will be shown in Remark 4.8) and makes the analytic calculation of $M^{*}$ and $m^{*}$ unfeasible, even when generally, $q(\omega)$ depends on $\omega$ through the norm $\|\omega\|^{2}$.

In the following, we show that under some special choices of either the generators or the spectral margins involved in (10) or (11), some interesting intervals for the parameter $\theta$ can be obtained, preserving the permissibility of the resulting structure.

Proposition 4.3 Let $\boldsymbol{g}=\boldsymbol{f}, \varphi_{i} \in \Phi_{\boldsymbol{f}}^{\mathcal{A}}\left(\right.$ resp. $\left.\varphi_{i} \in \Phi_{f}^{M}\right)$ for $i=1,2$, and $f_{\mathcal{A}}\left(\right.$ resp. $\left.f_{\mathrm{M}}\right)$ as defined in Eq. 10 (resp.(11)). Then:

(i) If $\varphi_{1} \circ \varphi_{2}^{-1}$ is subadditive (resp. convex), we have that $f_{\mathcal{A}}\left(\right.$ resp. $\left.f_{\mathrm{M}}\right)$ is a valid spectral density for any $\theta \in(-\infty, 1]$.

(ii) If $\varphi_{1} \circ \varphi_{2}^{-1}$ is superadditive (resp. concave), we have that $f_{\mathcal{A}}\left(\right.$ resp. $\left.f_{\mathrm{M}}\right)$ is a valid spectral density for any $\theta \in[0, \infty)$.

Proof Applying Corollary 3.2, for (i), the ordering $\mathcal{A}_{\varphi_{1}} \leq \mathcal{A}_{\varphi_{2}}$ implies $0 \leq m^{*} \leq M^{*} \leq 1$. Therefore, the optimal interval of Proposition 4.1 contains at least the interval $(-\infty, 1]$. For (ii), the reversed ordering leads to 

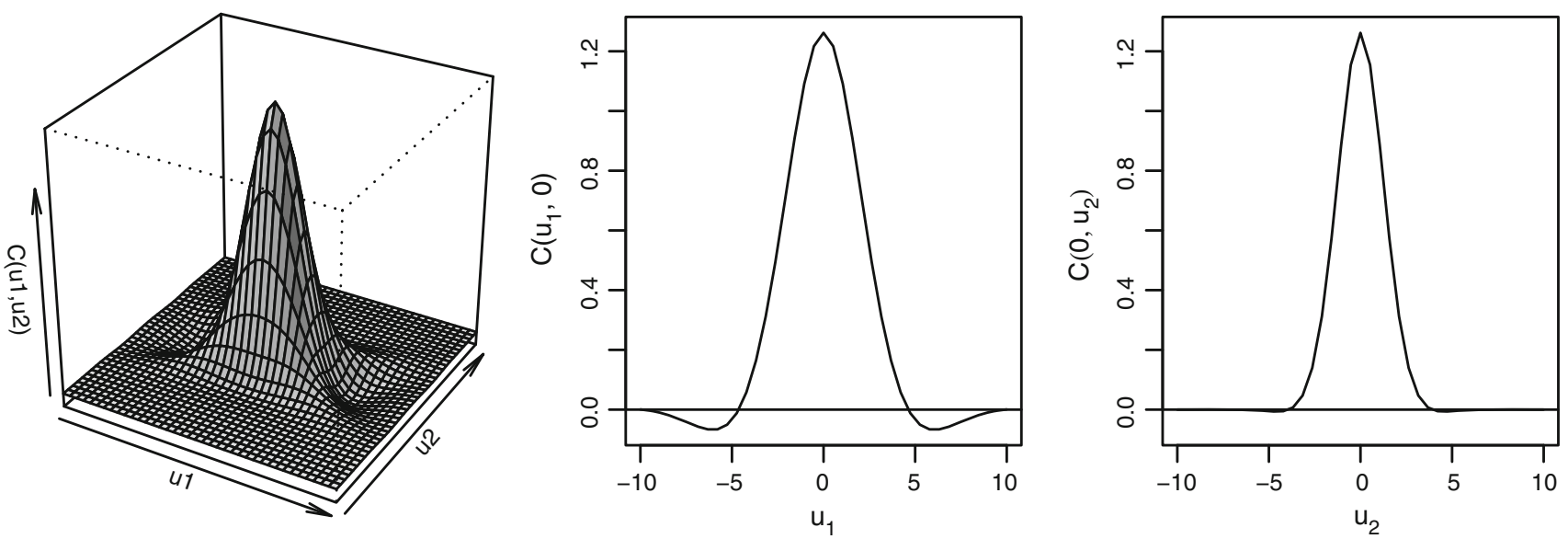

Fig. 1 Example of covariance function of Remark 4.5 obtained from the spectral density given by the linear combination $f\left(\omega_{1}, \omega_{2}\right)=5 \mathcal{A}_{\varphi_{1}}\left(f_{1}, f_{2}\right)\left(\omega_{1}, \omega_{2}\right)-4 \mathcal{A}_{\varphi_{1}}\left(f_{1}, f_{2}\right)\left(\omega_{1}, \omega_{2}\right)$, where $f_{1}(\omega)=f_{\text {Mat }}(\omega ; 1,5), f_{2}(\omega)=f_{\text {Mat }}(\omega ; 3,10), \varphi_{1}(t)=t^{-1}$ and $\varphi_{2}(t)=t^{-1 / 1.5}$

$1 \leq m^{*} \leq M^{*} \leq \infty$, which shows that the optimal interval contains at least the interval $[0, \infty)$.

Example 4.4 LPs. Let $d_{i}=1, i=1, \ldots, d$. For LPs, we can take any decreasing $\varphi \in \Phi$, independently of the vector of functions $\boldsymbol{f}$ representing the univariate margins. For instance, both the expressions

$$
\begin{aligned}
f_{\mathcal{A}}(\omega)= & \theta\left(\sum_{i=1}^{d} f_{i}\left(\omega_{i}\right)^{-\varepsilon_{1}}\right)^{-1 / \varepsilon_{1}} \\
& +(1-\theta)\left(\sum_{i=1}^{d} f_{i}\left(\omega_{i}\right)^{-\varepsilon_{2}}\right)^{-1 / \varepsilon_{2}}
\end{aligned}
$$

and

$$
\begin{aligned}
f_{M}(\omega)= & \theta\left(\sum_{i=1}^{d} \mathrm{w}_{\mathrm{i}} f_{i}\left(\omega_{i}\right)^{-\varepsilon_{1}}\right)^{-1 / \varepsilon_{1}} \\
& +(1-\theta)\left(\sum_{i=1}^{d} \mathrm{w}_{\mathrm{i}} f_{i}\left(\omega_{i}\right)^{-\varepsilon_{2}}\right)^{-1 / \varepsilon_{2}},
\end{aligned}
$$

for $\left(\mathrm{w}_{\mathrm{i}}\right)_{i=1}^{d}$ nonnegative weights summing up to one, can be seen as either parametric or semiparametric families of spectral densities indexed by the set $\Theta=\left\{\left(\theta, \varepsilon_{1}, \varepsilon_{2}\right)\right.$ : $\left.\theta \leq 1,0<\varepsilon_{1} \leq \varepsilon_{2}\right\}$, where the semiparametric construction would derive from the choice of nonparametric margins. Otherwise, the structure would be fully parametric. Similar comments apply to the structures

$f_{\mathcal{A}}(\omega)=\theta \prod_{i=1}^{d} f_{i}\left(\omega_{i}\right)+(1-\theta)\left(\sum_{i=1}^{d} f_{i}\left(\omega_{i}\right)^{-\varepsilon}\right)^{-1 / \varepsilon}$

and
$f_{M}(\omega)=\theta \prod_{i=1}^{d} f_{i}\left(\omega_{i}\right)^{\mathrm{w}_{i}}+(1-\theta)\left(\sum_{i=1}^{d} \mathrm{w}_{i} f_{i}\left(\omega_{i}\right)^{-\varepsilon}\right)^{-1 / \varepsilon}$

indexed by the parameter set $\Theta=\{(\theta, \varepsilon): \theta \geq 0, \varepsilon>0\}$, plus eventually the parameter set indexing the margins, whenever they are set to belong to a specified parametric family. The vector of functions $\left(f_{i}\right)_{i=1}^{d}$ can be taken to be a vector of different $\operatorname{ARMA}(p, q)$ spectral densities as presented in Sect. 2.

Remark 4.5 For the RF case, similar examples are valid, provided that the vector of spectral densities $\left(f_{i}\right)_{i=1}^{d}$ fulfills condition (A). For instance, taking each $f_{i}$ to be a univariate Matérn or a $\operatorname{AR}(p)$ spectral density. We show in Fig. 1 the plot of the resulting covariance function in $\mathbb{R}^{2}, C\left(u_{1}, u_{2}\right)$, and the two margins $C\left(u_{1}, 0\right)$ and $C\left(0, u_{2}\right)$, for the particular choice of $f_{1}(\omega)=f_{\text {Mat }}(\omega ; 1,5), f_{2}(\omega)=f_{\text {Mat }}(\omega ; 3,10)$, $\varphi_{1}(t)=t^{-1}$ and $\varphi_{2}(t)=t^{-1 / 1.5}$. The covariance function has been computed by direct discretization of the integral given by the Fourier transform as a Riemann sum.

Proposition 4.6 Let $\varphi_{1}=\varphi_{2}=\varphi \in \Phi_{f}^{\mathcal{A}} \cap \Phi_{g}^{\mathcal{A}} \quad$ (resp. $\left.\varphi \in \Phi_{f}^{M} \cap \Phi_{g}^{M}\right)$ such that $\mathcal{A}_{\varphi}$ (resp. $\left.M_{\varphi}\right)$ is a homogeneous operator. Then $f_{\mathcal{A}}$ (resp. $f_{M}$ ) as defined in Eq. 10 (resp. (11)) is a valid spectral density if

$$
\left[1-\max \left(1, \max _{i}\left(M_{i}\right)\right)\right]^{-1} \leq \theta \leq\left[1-\min \left(1, \min _{i}\left(m_{i}\right)\right)\right]^{-1} .
$$

Proof Let us show the result for the Archimedean case only, as the other case can be proved with the same arguments. Put $k_{1}=\min _{i} m_{i}$ and $k_{2}=\max _{i} M_{i}$. By the properties of $\varphi$, 


$\begin{array}{ccccc}k_{1} & \leq & q_{i}\left(\omega_{i}\right) & \leq & k_{2} \\ k_{1} g_{i}\left(\omega_{i}\right) & \leq & f_{i}\left(\omega_{i}\right) & \leq & k_{2} g_{i}\left(\omega_{i}\right) \\ \varphi\left(k_{1} g_{i}\left(\omega_{i}\right)\right) & \geq & \varphi\left(f_{i}\left(\omega_{i}\right)\right) & \geq & \varphi\left(k_{2} g_{i}\left(\omega_{i}\right)\right) \\ \sum_{i=1}^{k} \varphi\left(k_{1} g_{i}\left(\omega_{i}\right)\right) & \geq & \sum_{i=1}^{k} \varphi\left(f_{i}\left(\omega_{i}\right)\right) & \geq & \sum_{i=1}^{k} \varphi\left(k_{2} g_{i}\left(\omega_{i}\right)\right) \\ \varphi^{-1}\left(\sum_{i=1}^{k} \varphi\left(k_{1} g_{i}\left(\omega_{i}\right)\right)\right) & \leq & \varphi^{-1}\left(\sum_{i=1}^{k} \varphi\left(f_{i}\left(\omega_{i}\right)\right)\right) & \leq & \varphi^{-1}\left(\sum_{i=1}^{k} \varphi\left(k_{2} g_{i}\left(\omega_{i}\right)\right)\right) \\ \mathcal{A}_{\varphi}\left(k_{1} g\right)(\omega) & \leq & \mathcal{A}_{\varphi} f(\omega) & \leq & \mathcal{A}_{\varphi} k_{2}(\boldsymbol{g})(\omega) \\ k_{1} \mathcal{A}_{\varphi} \boldsymbol{g}(\omega) & \leq & \mathcal{A}_{\varphi} f(\omega) & \leq & k_{2} \mathcal{A}_{\varphi} \boldsymbol{g}(\omega) \\ k_{1}(\omega) & \leq & q(\omega) & \leq & k_{2} \\ k_{1} & \leq & m^{*} \leq M^{*} & \leq & k_{2}\end{array}$

Thus, the interval defined in Eq. 19 is a subinterval of the optimal interval (14), and $f$ shall be a valid spectral density whenever $\theta$ belongs to it.

Remark 4.7 If all $m_{i}$ and $M_{i}$ are, respectively, equal, the result is the complete characterization of the interval (i.e. necessary and sufficient condition for $f$ to be a valid spectrum).

Remark 4.8 When $\varphi(t)=-\log (t)$, i.e. in the tensor product case, the simple relations $m^{*}=\prod_{i=1}^{k} m_{i}, M^{*}=$ $\prod_{i=1}^{k} M_{i}$, allow to give the exact interval characterizing the valid spectral densities

$$
\left[1-\max \left(1, \prod_{i=1}^{k} M_{i}\right)\right]^{-1} \leq \theta \leq\left[1-\min \left(1, \prod_{i=1}^{k} m_{i}\right)\right]^{-1} .
$$

As a corollary of the previous results, we get the main results in Ma (2004) in a unified way, as it is stated subsequently.

Corollary 4.9 [Ma (2004), Thms. 1 and 2] For each $i=1,2, \ldots, d$, let $f_{i}\left(\cdot ; \alpha_{i}\right)$ be a parametric family of univariate spectral densities all defined on either $[0, \pi]$ or $\mathbb{R}$, let $\left(\alpha_{i}\right)_{i=1}^{d}$ and $\left(\beta_{i}\right)_{i=1}^{d}$ some fixed parameter vectors and let $f(\omega), \omega \in \Omega^{d}$ be the function defined in Eq. 2. Then:

(i) For $\Omega=[0, \pi]$, under the assumption

$$
\begin{aligned}
& \frac{f_{i}\left(0 ; \alpha_{i}\right)}{f_{i}\left(0 ; \beta_{i}\right)} \leq \frac{f_{i}\left(\omega ; \alpha_{i}\right)}{f_{i}\left(\omega ; \beta_{i}\right)} \leq \frac{f\left(\pi ; \alpha_{i}\right)}{f\left(\pi ; \beta_{i}\right)}, \quad \omega \in[0, \pi], \\
& \quad i=1,2, \ldots, d
\end{aligned}
$$

$f(\cdot)$ is a valid spectral density if and only if

$$
\begin{aligned}
& \left(1-\max \left(1, \prod_{i=1}^{d} \frac{f_{i}\left(\pi ; \alpha_{i}\right)}{f_{i}\left(\pi ; \beta_{i}\right)}\right)\right)^{-1} \\
& \quad \leq \theta \leq\left(1-\min \left(1, \prod_{i=1}^{d} \frac{f_{i}\left(0 ; \alpha_{i}\right)}{f_{i}\left(0 ; \beta_{i}\right)}\right)\right)^{-1} .
\end{aligned}
$$

(ii) For $\Omega=\mathbb{R}$, under the assumptions $\lim _{\omega \rightarrow \infty} \omega^{2 p_{i}} f_{i}\left(\omega ; \alpha_{i}\right)=\frac{1}{f_{i}\left(0 ; \alpha_{i}\right)}, \lim _{\omega \rightarrow \infty} \omega^{2 p_{i}} f_{i}\left(\omega ; \beta_{i}\right)=\frac{1}{f_{i}\left(0 ; \beta_{i}\right)}$

for some $p_{i}>0$, and

$\frac{f_{i}\left(0 ; \beta_{i}\right)}{f_{i}\left(0 ; \alpha_{i}\right)} \leq \frac{f\left(\omega ; \alpha_{i}\right)}{f\left(\omega ; \beta_{i}\right)} \leq \frac{f_{i}\left(0 ; \alpha_{i}\right)}{f_{i}\left(0 ; \beta_{i}\right)}, \quad \omega \in[0, \infty)$,

$i=1,2, \ldots, d$,

$f(\cdot)$ is a valid spectral density if and only if

$$
\left(1-\prod_{i=1}^{d} \frac{f_{i}\left(0 ; \alpha_{i}\right)}{f_{i}\left(0 ; \beta_{i}\right)}\right)^{-1} \leq \theta \leq\left(1-\prod_{i=1}^{d} \frac{f_{i}\left(0 ; \beta_{i}\right)}{f_{i}\left(0 ; \alpha_{i}\right)}\right)^{-1} .
$$

Proof Obviously, the assumptions in each case imply, on the one hand, that $m_{i}=q_{i}(0)$ and $M_{i}=q_{i}(\pi)$, and on the other hand, that $m_{i}=\lim _{\|\omega\| \rightarrow \infty} q_{i}(\omega)$ and $M_{i}=q_{i}(0)$. Also notice that the assumptions on the RF case imply $m_{i} \leq 1 \leq M_{i}=m_{i}^{-1}$ for each $i$.

Remark 4.10 Property (i) is satisfied, for instance, by the class of spectra associated to AR processes on the real line as in Eq. 3, whilst property (ii) is satisfied for instance by the Matérn class, as shown by Ma (2004).

Example 4.11 LPs The expressions

$$
\begin{aligned}
f_{\mathcal{A}}(\omega)= & \theta\left(\sum_{i=1}^{k} f_{i}\left(\omega_{i}\right)^{-\varepsilon}\right)^{-1 / \varepsilon}+(1 \\
& -\theta)\left(\sum_{i=1}^{k} g_{i}\left(\omega_{i}\right)^{-\varepsilon}\right)^{-1 / \varepsilon}
\end{aligned}
$$

and

$$
\begin{aligned}
f_{M}(\omega)= & \theta\left(\sum_{i=1}^{k} \mathrm{w}_{\mathrm{i}} f_{i}\left(\omega_{i}\right)^{-\varepsilon}\right)^{-1 / \varepsilon}+(1 \\
& -\theta)\left(\sum_{i=1}^{k} \mathrm{w}_{\mathrm{i}} g_{i}\left(\omega_{i}\right)^{-\varepsilon}\right)^{-1 / \varepsilon}
\end{aligned}
$$

can be seen as parametric families of spectral densities for 
LPs indexed by the set $\Theta=\left\{(\theta, \varepsilon): \theta_{m} \leq \theta \leq \theta_{M}, \varepsilon>0\right\}$, where

$\theta_{m}=\left[1-\max \left(1, \max _{i}\left(M_{i}\right)\right)\right]^{-1}$,

$\theta_{M}=\left[1-\min \left(1, \min _{i}\left(m_{i}\right)\right)\right]^{-1}$.

Based on the fact that the maximum, minimum and geometric means are limiting cases of $\varphi$-means, we can also state that the expressions

$f_{\mathcal{A}}(\omega)=\theta \prod_{i=1}^{k} f_{i}\left(\omega_{i}\right)+(1-\theta) \prod_{i=1}^{k} g_{i}\left(\omega_{i}\right)$,

and

$f_{M}(\omega)=\theta \prod_{i=1}^{k} \mathrm{w}_{i} f_{i}\left(\omega_{i}\right)+(1-\theta) \prod_{i=1}^{k} \mathrm{w}_{i} g_{i}\left(\omega_{i}\right)$,

$f_{M}(\omega)=\theta \min _{i} f_{i}\left(\omega_{i}\right)+(1-\theta) \min _{i} g_{i}\left(\omega_{i}\right)$,

$f_{M}(\omega)=\theta \max _{i} f_{i}\left(\omega_{i}\right)+(1-\theta) \max _{i} g_{i}\left(\omega_{i}\right)$,

can be seen as parametric families of spectral densities of LPs indexed by the set $\Theta=\left\{\theta: \theta_{m} \leq \theta \leq \theta_{M}\right\}$.

Consider univariate margins of the type $f_{i}(\cdot)=$ $f_{\mathrm{ARMA}}\left(\cdot ; \alpha_{i}\right)$ and $g_{i}(\cdot)=g_{\mathrm{ARMA}}\left(\cdot ; \beta_{i}\right),-1<\alpha_{i}, \beta_{i}<1, i=$ $1, \ldots, k$. One can show that

$\theta_{m}=\left[1-\max \left(\frac{1-\beta_{i}}{1-\alpha_{i}}, \frac{1+\beta_{i}}{1+\alpha_{i}}\right)^{2}\right]^{-1}$,

and

$\theta_{M}=\left[1-\min \left(\frac{1-\beta_{i}}{1-\alpha_{i}}, \frac{1+\beta_{i}}{1+\alpha_{i}}\right)^{2}\right]^{-1}$.

RFs Let $f_{i}(\cdot)=f_{\text {Mat }}\left(\cdot ; \alpha_{i}, v\right)$ and $g_{i}(\cdot)=f_{\text {Mat }}\left(\cdot ; \beta_{i}, v\right)$, with $v>0$ and $\alpha_{i}, \beta_{i}>0$ for each $i=1, \ldots, d$, we get

$\begin{aligned} \theta_{m} & =\left[1-\left(\frac{\max _{i}\left(\alpha_{i}, \beta_{i}\right)}{\min _{i}\left(\alpha_{i}, \beta_{i}\right)}\right)^{d_{i} / 2}\right]^{-1}, \\ \theta_{M} & =\left[1-\left(\frac{\min _{i}\left(\alpha_{i}, \beta_{i}\right)}{\max _{i}\left(\alpha_{i}, \beta_{i}\right)}\right)^{2 v}\right]^{-1} .\end{aligned}$

Another interesting case is obtained by setting $f_{i}(\cdot)=$ $f_{\mathrm{AR}}\left(\cdot ; \alpha_{i}\right)$ and $g_{i}(\cdot)=f_{\mathrm{AR}}\left(\cdot ; \beta_{i}\right)$, with $\alpha_{i}=\left(\alpha_{j i}\right)_{j=1}^{p}, \beta_{i}=$ $\left(\beta_{j i}\right)_{j=1}^{p}, \alpha_{i}, \beta_{i} \subset(0, \infty)^{p}$ where, for each $i \in\{1, \ldots, d\}$, either the order $\alpha_{j i} \leq \beta_{j i}$ or $\alpha_{j i}>\beta_{j i}$ holds for all $j \in\{1, \ldots, p\}$. In this case we obtain

$$
\begin{aligned}
\theta_{m} & =\left[1-\max _{i}\left(\prod_{j=1}^{p} \frac{\max _{j}\left(\alpha_{j i}, \beta_{j i}\right)}{\min _{j}\left(\alpha_{j i}, \beta_{j i}\right)}\right)\right]^{-1}, \\
\theta_{M} & =\left[1-\min _{i}\left(\prod_{j=1}^{p} \frac{\min _{j}\left(\alpha_{j i}, \beta_{j i}\right)}{\max _{j}\left(\alpha_{j i}, \beta_{j i}\right)}\right)\right]^{-1} .
\end{aligned}
$$

\section{Conclusions}

The main novelty of this work is the construction of new $d$ dimensional lattice and RF models based on a special mixing of models in lower dimensions (simple models), being the $d$-dimensional domain of the new model the product of the domains of the simple models.

The actual literature on nonseparable covariance functions is rather limited in terms of flexible functions. In data fitting practice, one first tests for separability (space vs time or within spatial coordinates). If this hypothesis is rejected then looks for a particular function within the family of nonseparable functions. To our knowledge, the existing functions are quite rigid and complicated for the practical analysis. A positive point of our proposal is that we start from simple marginals (simple in both the analytical expression and the way to use in practice), and more complicated structures are built upon them. These new structures are in general more flexible and clearly enlarge the current number of families. For instance, we propose to perform data fitting in $\mathbb{R}^{2}$ by using the Matérn bivariate family. If the resulting function satisfies a good fit the work is done. Otherwise, we propose the use of an Archimedean composition and/or a quasi-arithmetic mean of two members of the univariate Matérn family, with a simple parametric family of $\varphi$. The techniques described herein are also useful for building new classes of $d$-dimensional models starting from models on a common $d$-dimensional domain. Let us make an abuse of notation and write

$$
\begin{aligned}
& \mathcal{A}_{\varphi} f(\omega)=\varphi^{-1}\left(\sum_{i=1}^{k} \varphi\left(f_{i}(\omega)\right)\right), \\
& M_{\varphi} f(\omega)=\varphi^{-1}\left(\sum_{i=1}^{k} \mathrm{w}_{i} \varphi\left(f_{i}(\omega)\right)\right)
\end{aligned}
$$

for $\boldsymbol{f} \in \mathcal{F}$. Permissibility conditions are rather trivial now: the upper bounds $\sum_{i} f_{i}$ (for the Archimedean composition whenever $\varphi^{-1}$ is superadditive) and $\max _{i} f_{i}$ (for the quasi arithmetic mean) are now integrable in $\Omega^{d}$ for both the lattice and the RF case. In the study of the linear combinations with eventually negative weights, similar results are obtained, leading to (non simple but) potentially applicable examples based on combinations of $d$-dimensional Matérn or tensor products of $\operatorname{AR}(p)$ or $\operatorname{ARMA}(p, q)$ spectral densities. In this spirit, Porcu et al. (2008) made 
Archimedean compositions of two spectral densities, through exclusively completely monotone generators, as a tool in order to enlarge the family of nonstationary covariance functions created by Pintore and Holmes (2010) using the spatial adaption technique. In this paper, we have set the solid background for that work and others, either theoretical or practical ones, that will be developed in the time to come in the field of lattice processes and RFs.

Acknowledgements This study was initiated when Emilio Porcu was research fellow at the Universitat Jaume I, department of Mathematics. He also acknowledges the support of the research fund FOR-916 Statistical Regularization. Jorge Mateu and Pablo Gregori acknowledge the support of MTM2010-14961 from the Spanish Ministry of Science and Education.

\section{References}

Besag JE (1974) Spatial interaction and the statistical analysis of lattice systems (with discussion). J R Stat Soc B 36:192-236

Bochner S (1933) Monotone funktionen, stiltjes integrale und harmonische analyse. Math Ann 108:378-410

Cauchy AL (1821) Cours d'analyse de l'Ecole Royale Polytechnique, Vol. I. Analyse algébrique, Debure, Paris

Chisini O (1929) Sul concetto di media. Periodico di Matematiche 9(4):106-116

Christakos G (1991) A theory of spatiotemporal random fields and its application to space-time data processing. IEEE Trans Syst Man Cybern 21(4):861-875

Christakos G (1992) Random field models in earth sciences. Academic Press, San Diego, p 474

Fuentes M (2002) Interpolation of nonstationary air pollution processes: a spatial spectral approach. Stat Model 2:281-298

Fuentes M (2005) A formal test for nonstationarity of spatial stochastic processes. J Multivar Anal 96:30-54

Fuentes M (2006) Testing for separability of spatial-temporal covariance functions. J Stat Plan Inference 136:447-466

Genest C (1987) Frank's family of bivariate distributions. Biometrika 74:549-555

Genest C, MacKay RJ (1986) Copules archimédiennes et familles de lois bidimensionnelles dont les marges sont données. Revue Canadienne de Statistique 14(2):145-149

Gneiting T (2002) Stationary covariance functions for space-time data. J Am Stat Assoc 97:590-600

Gneiting T, Genton MG, Guttorp P (2007) Geostatistical space-time models, stationarity, separability and full symmetry. In: Finkenstadt B, Held L, Isham V (eds) Statistical methods for spatiotemporal systems, Chapman \& Hall/CRC, Boca Raton, pp 151-175

Gregori P, Porcu E, Mateu J, Sasvári Z (2008) On potentially negative space time covariances obtained as sum of products of marginal ones. Ann Inst Stat Math 60:865-882

Hardy GH, Littlewood JE, Pólya G (1934) Inequalities. Cambridge Univ. Press, Cambridge

Janauer GA (2001) Is what has been measured of any direct relevance to the success of the macrophyte in its particular environment? In: Ravera $\mathrm{O}$ (ed) Scientific and legal aspects of biological monitoring in freshwater, J Limnol 60 (Suppl 1):33-38

Kolmogorov AN (1930) Sur la notion de la moyenne. Atti Accad Naz Lincei Mem Cl Sci Fis Mat Natur Sez 12(6):388-391
Ma C (2004) A class of stationary random fields with a simple correlation structure. J Multivar Anal 94(2):313-327

Marichal J-L (2000) On an axiomatization of the quasi-arithmetic mean values without the symmetry axiom. Aequationes Mathematicae 59(1-2):74-83

Martin RJ (1996) Some results on unilateral ARMA lattice processes. J Stat Plan Inference 50:395-411

Matérn B (1960) Spatial variation: stochastic models and their application to some problems in forest surveys and other sampling investigations. Meddelanden Fran Statens Skogsforskningsinstitut, Band 49, 5, Stockholm

Matheron G (1989) Estimating and choosing. Springer, Berlin

Nagumo M (1930) Ober eine klasse der mittelwerte. Jap J Math 7:71-79

Ostoja-Starzewski M (2002) Microstructural randomness versus representative volume element in thermomechanics. ASME J Appl Mech 69:25-35

Ostoja-Starzewski M (2007) Towards thermomechanics of fractal media. J Appl Math Phys 58:1085-1096

Ostoja-Starzewski M (2008) Microstructural randomness and scaling in mechanics of materials. Chapman \& Hall/CRC Press

Pintore A, Holmes CC (2010) Non-stationary covariance functions via spatially adaptive spectra. "Tentatively accepted" J Am Stat Assoc (in press)

Pomeroy JW, Toth B, Granger RJ, Hedstrom NR, Essery RLH (2003) Variation in surface energetics during snowmelt in a subarctic mountain catchment. J Hydrometeorol 4:702-719

Porcu E, Gregori P, Mateu J (2006) Nonseparable stationary anisotropic space-time covariance functions. Stoch Env Res Risk Assess 21(2):113-122

Porcu E, Mateu J, Saura F (2008) New classes of covariance and spectral density functions for spatio-temporal modelling. Stoch Env Res Risk Assess 22(suppl.1):65-79

Porcu E, Gregori P, Mateu J (2009) Archimedean spectral densities for nonstationary space-time Geostatistics. Statistica Sinica 19(1): 273-286

Ruiz-Medina MD, Angulo JM (2002) Spatio-temporal filtering using wavelets. Stoch Env Res Risk Assess 16(4):241-266

Rytov SM, Kravtsov YA, Tatarskii VI (1989) Principles of statistical radiophysics, vol 4. Springer, Berlin

Scaccia L, Martin RJ (2005) Testing axial symmetry and separability of lattice processes. J Stat Plan Inference 131(1):19-39

Shkarofsky IP (1968) Generalized turbulence space-correlation and wave-number spectrum-function pairs. Can J Phys 46:2133-2140

Stein ML (1995) Fixed-domain asymptotics for spatial periodograms. J Am Stat Assoc 90:1277-1288

Stein ML (1999) Shape interpolation of spatial data. Some theory of kriging. Springer, New York

Stein ML (2005) Space-time covariance functions. J Am Stat Assoc 100:310-321

Whittle P (1954) On stationary processes in the plane. Biometrika 41:434-449

Yadrenko M (1983) Spectral theory of random fields. Optimization Software, New York

Yu H-L, Kolovos A, Christakos G, Chen J-C, Warmerdam S (2006) Interactive spatiotemporal modelling of health systems: the SEKS-GUI framework. Stoch Env Res Risk Assess 21(5):555-572

Zhang H (2004) Inconsistent estimation and asymptotically equal interpolations in model-based geostatistics. J Am Stat Assoc 99:250-261 